\title{
Machining Surface Quality Analysis of Aluminum Alloy Thin-Walled Parts in Aerospace
}

\author{
${ }^{1}$ Dai Bing, ${ }^{1} Y u$ Guang-bin, ${ }^{1}$ Guan Yan-qi, ${ }^{1}$ Shao Jun-peng, \\ ${ }^{1}$ Guan Yan-qi, ${ }^{2}$ Wu Xue-mei, ${ }^{3}$ Liu Yu-xin \\ ${ }^{1}$ School of Mechanical and Power Engineering, \\ Harbin University of Science and Technology, \\ Harbin, Heilongjiang 150080, China \\ ${ }^{2}$ School of Mechanical Electrical Engineering, \\ Harbin Institute of Technology, harbin 150001, China \\ ${ }^{3}$ Harbin Hafei Industry CO., LTD harbin 150066, China
}

\begin{abstract}
There are many key parts in the field of aerospace with aluminum alloy thin-walled parts, the problem of breaking the low efficiency in production that's also followed. There will be a lot of deformation in processing because of its poor rigidity, poor process, which will lead to low processing efficiency. It is of great significance to improve the machining efficiency of the aluminum alloy thin-walled parts, and a series of analysis of the surface quality of aluminum alloy thin-walled parts there are carried out clamping, cutting tools, take the knife route so on, and the problems of low machining efficiency and poor surface quality are solved.
\end{abstract}

Keywords: thin-walled parts, Take the knife route, Machined surface

\section{Introduction}

With the continuous progress of science and technology, the requirements of the quality of thin wall parts in aircraft are also continuously improving, especially for aluminum alloy thin walled parts. It has a wide range of applications in many areas because of its unique high strength, low density, corrosion resistance and load carrying capacity, etc[1]..Thin wall parts generally refers to the wall thickness and the size of the proportion of less than 1/20 of the parts, parts of the shape is more complex, the structure is low, processing margin. In order to solve this problem, it is usually to reduce or inhibit the deformation of parts by cutting down the amount of cutting to process, and combining with the corresponding manual correction method to compensate, this method not only makes the productivity significantly decreased, but also difficult to control the processing quality. Throughout the factors that cause the thin wall parts in the process of deformation, including the material removal process of heat, cutting force, vibration and clamping force, etc.. In the milling process, these factors are usually comprehensive affect ,and forming the final deformation of the processing parts [2].

In this paper, the influence of the residual stress distribution, processing plan, the assembly plan, the selection of tool and cutting route on the machining quality of the workpiece is analyzed, and the machining process of the thin-walled parts is optimized to improve the machining quality of thin-walled parts, and it has certain guiding significance in practice. 


\section{The Main Reason of Machining Deformation of Thin-Walled Parts}

General aluminum alloy thin wall parts of the processing method of the use of milling, the mechanical processing of the more difficult, a lot of factors will lead to deformation, for this reason, this paper carried out the following analysis.

The elastic modulus of aluminum alloy is 70GPa, its elastic modulus is small, and its structure stiffness is small, so it is easy to produce the elastic deformation of the workpiece when machining aluminum alloy. Therefore, under the same condition, the deformation of the workpiece in the aluminum alloy is larger than that of the same structure of steel [3]. In addition, in the process, the residual stress in the blank is not destroyed. When machining, the part of the material is removed and the residual stress is released. When the thin wall parts are cut, the cutting force and cutting heat generated during machining, the stress distribution in the workpiece, and the deformation of the parts. For aluminium alloy thin wall parts, clamping force can cause part deformation. Because of its poor rigidity, the deformation caused by the loading can affect the position, shape and size of the surface, so the clamping force is an important factor to cause the workpiece deformation. In cutting process, the interaction between the clamping force and the residual stress in the cutting force, resulting in the deformation of the workpiece under the coupling of the machining residual stress and the initial residual stress. The cutting line is one of the factors that affect the deformation of thin wall parts. The different direction and size of the original residual stress release in the blanks are different. The internal residual stress and the residual stress in the workpiece are changed gradually, and the deformation of the workpiece is changed.

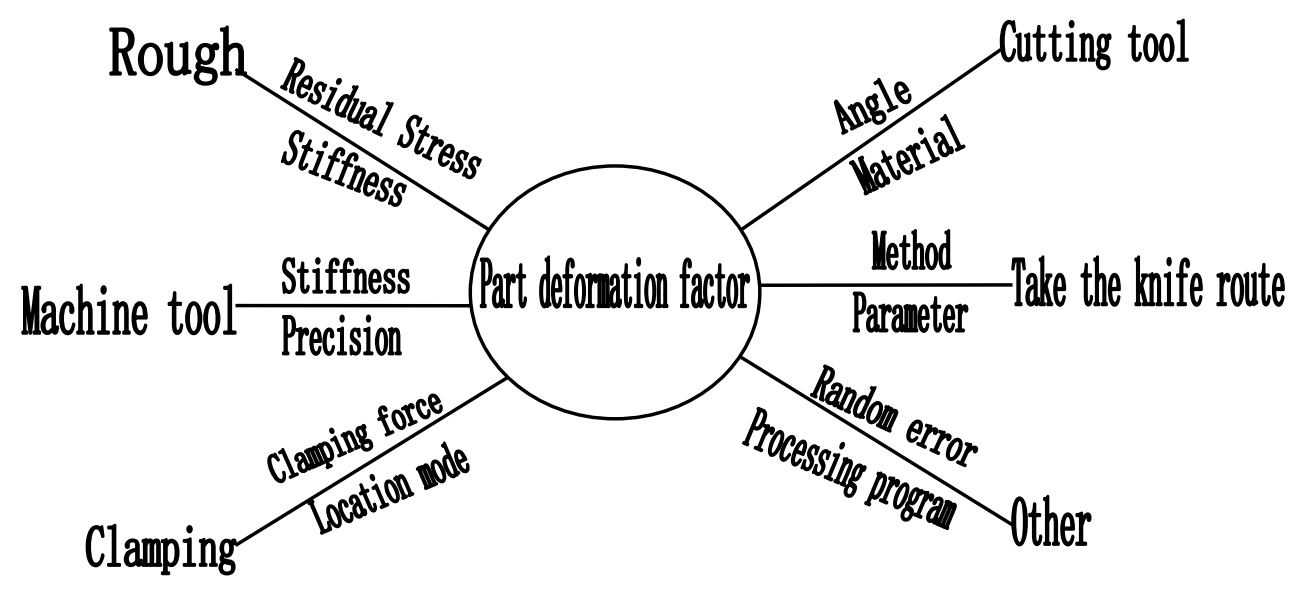

Figure 1. Deformation Factor of Thin-Walled Parts

\section{Improvement Measures for Machining Process of Thin-Walled Parts}

The general steps of the thin wall parts processing can be divided into clamping, cutting tools, cutting line, rough machining, finishing, and so on, where the design of each step is related to the size of the workpiece deformation, so every step should be taken seriously.

\subsection{Improvement of Process Plan}

In the process of aluminum alloy thin wall parts, the design of the part machining scheme is directly influenced by the effective control of the machining deformation. It 
should reduce the clamping force and cutting force, in order to work smoothly, it should be reasonable design fixture, so that the clamping force can not be too small, otherwise it will form a larger vibration in the processing, clamping force should be close to the cutting force generated by the maximum cutting force. At the same time, according to the requirements of machining accuracy, the accuracy of aluminum alloy thin wall parts, the more the number of the processing, the general application of rough machining - semi finishing - finishing process plan [4].

Workpiece in rough machining should adopt the larger clamping force and cutting amount as soon as possible to remove the material, both positive and negative sides to remove the number according to the actual situation and set, the processing is based on the back of the benchmark, the use of symmetrical milling method can maximize the reduction because of the cumulative deformation, rough machining should set reasonable cutting margin to maintain the processing quality. When the workpiece is finished, it is necessary to arrange the effective heat treatment to eliminate the residual stress during machining process, which can improve the stability of the workpiece, and lay a good foundation for the next process. In order to maintain machining accuracy in a certain range, it should be possible to reduce the cutting force and feed rate, so that the workpiece can not produce larger deformation, and to ensure that the workpiece machining is stable, the clamping force should be selected to reduce the clamping deformation, and the aluminum alloy thin wall parts can be better guaranteed[5].

Process scheme takes into account changes in the stiffness of the machining process, try to increase the stiffness of the parts in the machining process is, which increases the machining method has side adding ribbed slab, paraffin or rubber mud filling to the parts and so on. In the actual process, according to the actual conditions and the structural characteristics of the parts should be taken into account the part of the processing method and then develop a thin-walled parts of the processing program to achieve at least the processing deformation.

\subsection{Optimization of Clamping Positioning Scheme}

Aerospace Thin-walled Parts in the machining process, the machining deformation caused by many factors, including clamping due to deformation caused by accounting for $20 \%-60 \%$ So the assembly of the thin-walled parts is very important, it can greatly affect the degree of deformation of the workpiece. According to the structural characteristics and processing mode, the overall stress and deformation analysis of the thin wall parts, especially to the reasonable selection of positioning, fixture and fixture layout, so as to maintain the stability of workpiece machining, and to increase the process rigidity. In the design of thin walled pieces of clamping plan should follow the following aspects:

(1) a reasonable choice of clamping form, the positioning surface should be selected as the workpiece a large area of flat or curved surface, the workpiece and the workpiece positioning contact area should be as large as possible, in order to improve contact stiffness, to prevent deformation.

(2) the thin wall parts should be avoided when clamping force is not average, and the number of clamping points is not too small, otherwise it will cause the workpiece deformation, resulting in the workpiece obsolete ; the clamping force of the general thin-walled parts in a rigid good surface, to prevent the workpiece deformation.

(3) if the thin-walled parts of the processing are symmetrical, the symmetry of the clamping scheme should be adopted. 


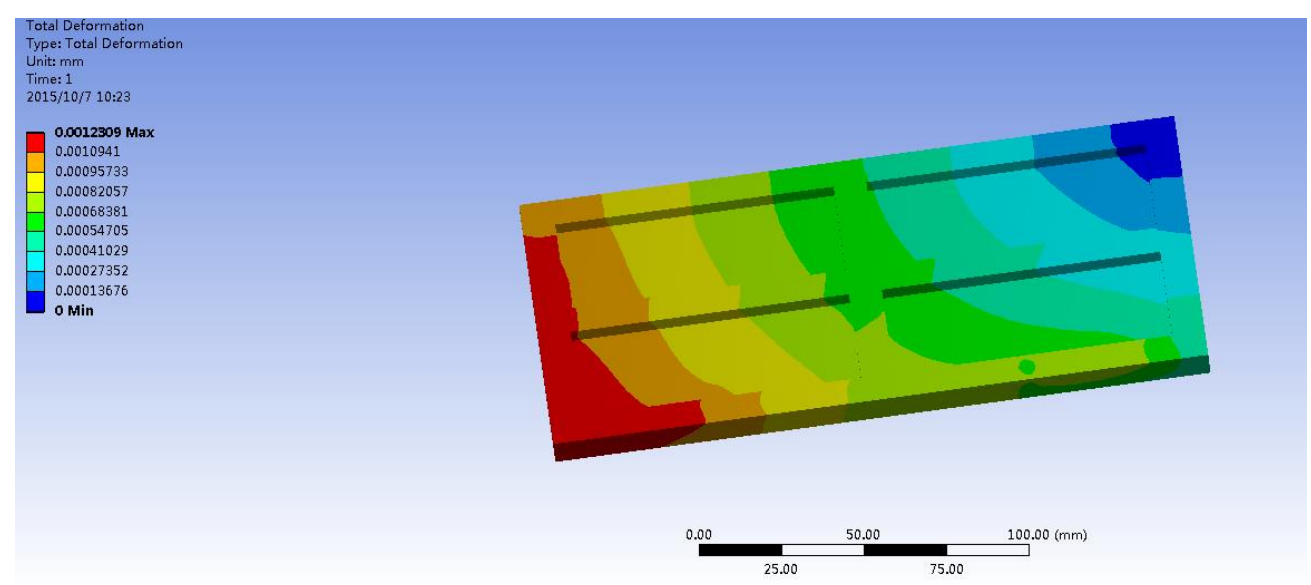

(a) Total Deformation Analysis of the Clamping Plate Scheme

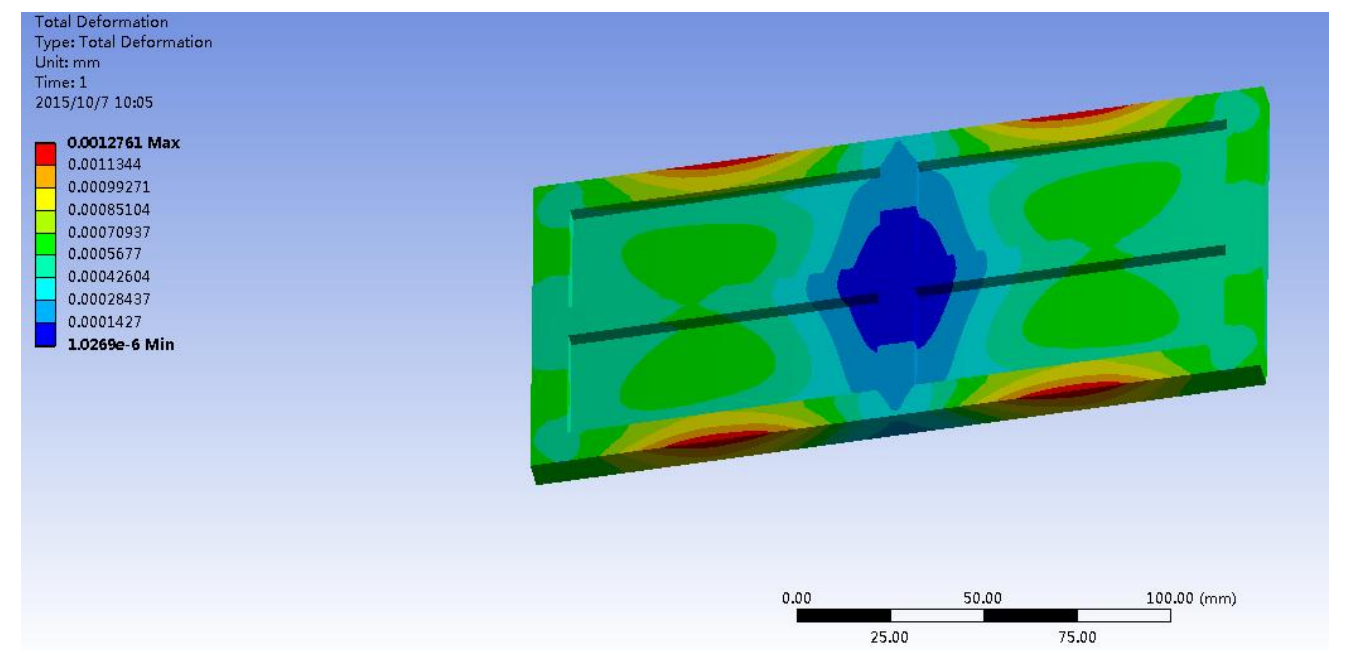

(b) Total Deformation Analysis Figure of Combined Fixture Scheme

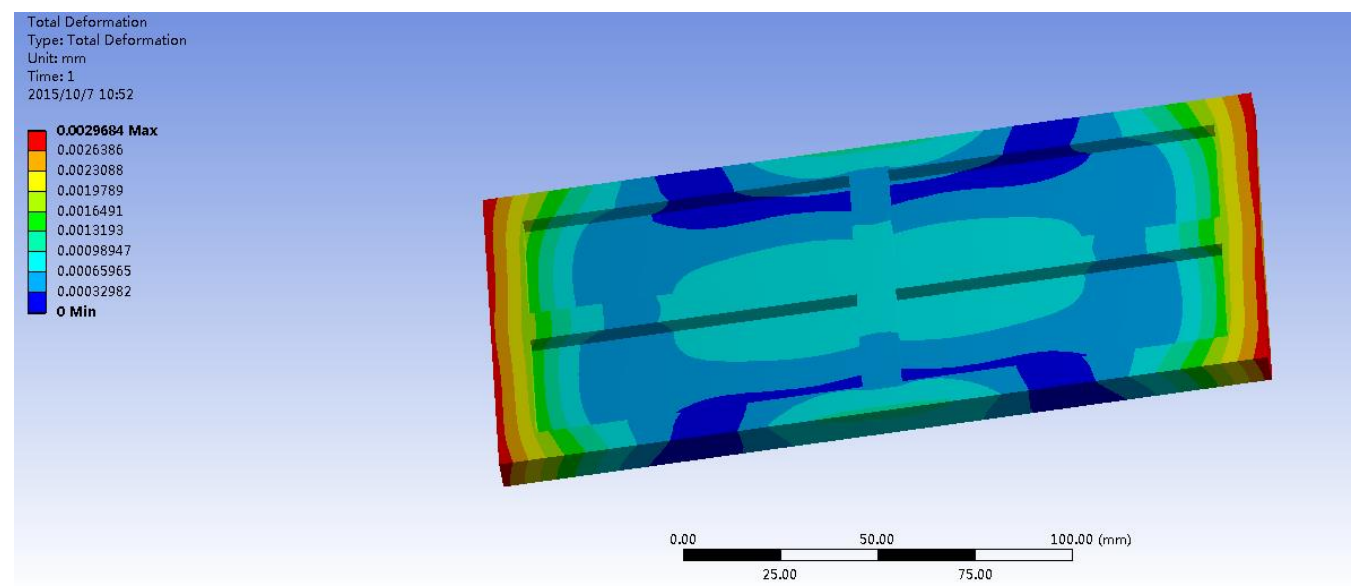

(c) Total Deformation Analysis of the Bolt Clamping Scheme 


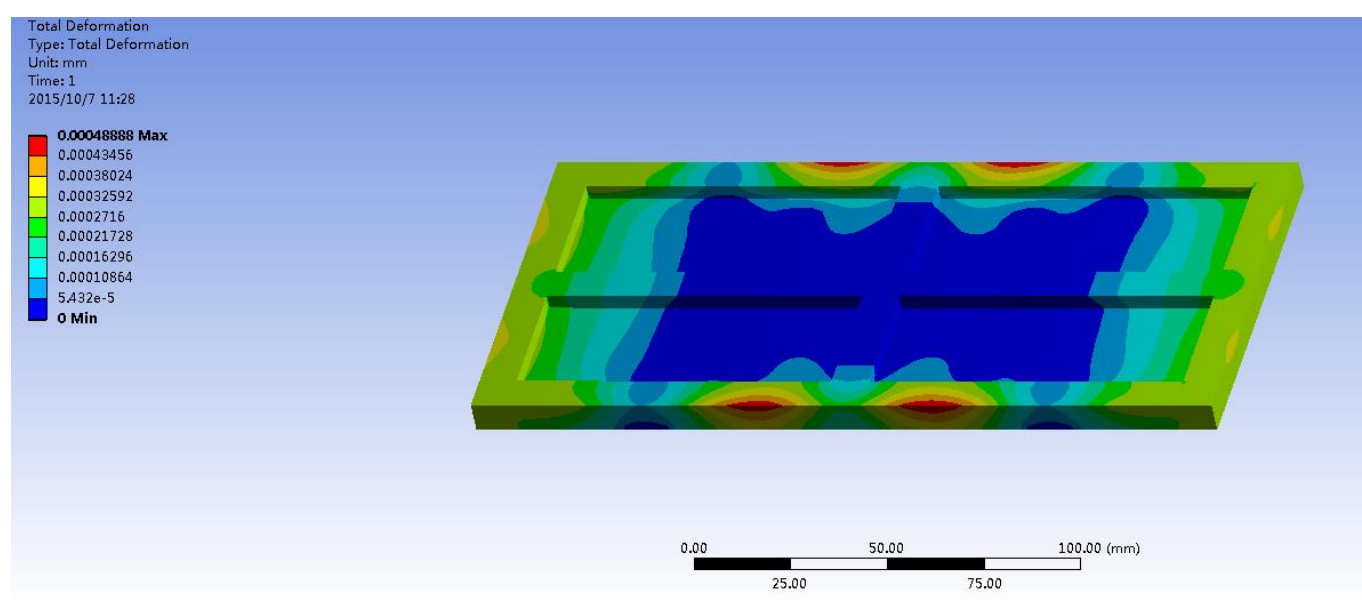

(d) Total Deformation Analysis of the Glue Clamping Scheme

\section{Figure 2. Total Deformation of the Clamping Scheme}

From the thin-walled parts machining scheme we know that in rough machining of the workpiece after the completion of the required finish machining, the effect in the process of clamping force is very important. According to the shape of parts, this paper designed several random clamping schemes and analysis of ANSYS Workbench 15 as shown in Figure 2 .

Table 1. The Maximum Deformation of the Clamping Plan

\begin{tabular}{c|c|c|c|c}
\hline $\begin{array}{c}\text { Clamping } \\
\text { scheme }\end{array}$ & Clamp plate & $\begin{array}{c}\text { Combined } \\
\text { fixture }\end{array}$ & Bolt clamping & $\begin{array}{c}\text { Glue } \\
\text { clamping }\end{array}$ \\
\hline $\begin{array}{c}\text { Maximum } \\
\text { deformation }\end{array}$ & $1.23 \mathrm{um}$ & $1.28 \mathrm{um}$ & $2.97 \mathrm{um}$ & $0.49 \mathrm{um}$ \\
\hline
\end{tabular}

According to Table 1, we can know that the total deformation of the glue clamping is the smallest, so the method of the glue clamping is used for the assembly.

\subsection{Cutter's SelectionScheme}

The difference of the residual stress distribution is different, so the different parts have different matching tools and cutting conditions, so as to achieve good cutting results and improve the cutting efficiency, cutting tool material should have excellent high temperature mechanical properties, high heat resistance, high thermal shock resistance and high reliability. Processing of aluminum alloy thin-walled parts, general selection of tool materials have hard alloy, ceramics, cubic boron carbide, polycrystalline diamond, etc[6].

Selection of cutting tool angle in milling aluminum alloy thin walled parts:

(1) Rake angle angle between the rake face and the base angle, its size directly affects the strength of the blade, rake angle can not be too small, otherwise it will increase before the knife cutting pressing surface layer deformation, cut the chip flow resistance is large, the rake face will aggravate the wear and cutting tool cutting force greatly, reduce the service life of the tool; but the angle degree is too large, blade 
cooling will become slow and the strength of the tool will also reduce the so the tool will aggravate the wear and chipping.

(2) The selection of rear corner cutting tool will affect the tool stiffness. The main function is to reduce the friction between the tool and the machined surface, in order to reduce the friction between the blank and the cutting tool, the choice of the rear corner is not too small. Too small the rear corner will reduce the tool life, but when the angle is too large will make the intensity of the blade is poor, while the heat dissipation conditions will become worse, but will aggravate the wear and tear. Therefore, we must consider the comprehensive factors, select the appropriate angle.

(3) The main effect of the angle of the cutting edge is to make the cutting edge cut and cut out the workpiece and control the direction of the chip flow and improve the stability of the process. Because the aluminum alloy material with a certain plastic, chip morphology is the strip, so the choice of the angle of the blade angle should be slightly larger. When the hard aluminum alloy in high speed milling, the angle of the cutting edge of the general use of 20 degrees $~ 25$ degrees, if less than 20 degrees, will make the deformation of chip increases, while the surface quality of the machined surface.

Finally, the choice of tool teeth number should be moderate, cutter tooth number is too small will reduce the tool's stiffness and cross-sectional area, but the number of teeth is too much, when the cutter tooth number is too much, cutting tool for cutting the space becomes small, so that the chip is not easy to discharge, resulting in cutting plug, while the surface of the cold work, resulting in the service life of the cutting tool [7].

\subsection{Take the Knife Route}

In the processing of thin-walled parts, the cutting force of the cutter is the factor that affects the deformation, the cutting force and the degree of transmission of the workpiece in machining process are different. Therefore, the distribution of the residual stress of the thin-walled parts is determined by the cutter route.

The influence of the cutting line on the residual stress is mainly as follows:

(1) The different cutting force of different paths is different, which causes different deformation of the workpiece.

(2) Different ways of removing the material are also different. The initial residual stress of the workpiece is released along with the material removal, and the direction of the stress release is determined by the knife line. The cutter selection is reasonable and the surface quality of thin wall parts [8].

Firstly, feeding mode selection is very important, to avoid vertical knife, when using this method, tools will be on the surface of the workpiece and leave scratches, reduce the surface accuracy, as far as possible the use of screw feed or arc feed to ensure the tool path smoothing. Feed the tool should be slow to cut into the workpiece, as smooth as possible, this tool on the workpiece surface does not leave obvious traces, and high surface precision. 


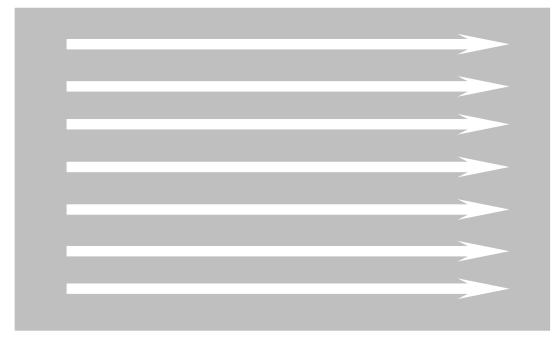

(a) Simultaneously Milling

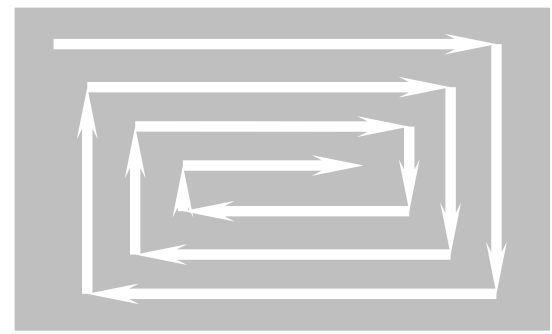

(c) From Outside to Inside Milling

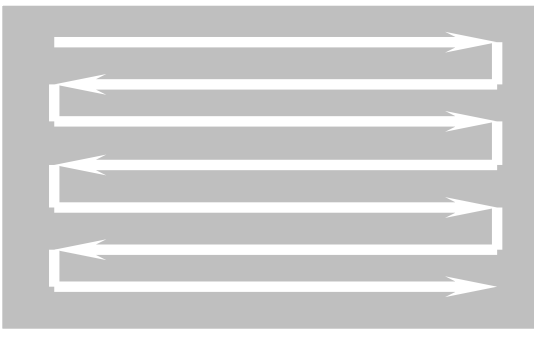

( b)The "S" Type Milling

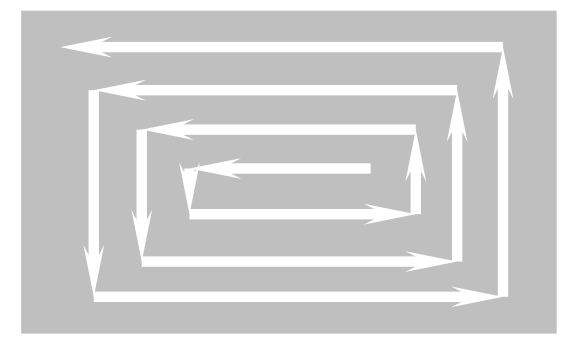

(d) From Inside to Outside Milling

Figure 3. The Route of Knife

Secondly, choose the right to take the knife route, the thin wall parts processing of the knife line is generally common in four types, as shown in Figure 3, the type of milling, and into the milling, from the outside to the inside and outside the milling. Through the theoretical analysis of [9], in order to ensure the other processing conditions, only to change the situation of the knife line, the degree of deformation of thin-walled parts has a big difference. Which is processed by the outside of the milling process in the form of small deformation, so in the processing of aluminum alloy thin wall parts can be used by the outgoing internal milling. From outside to inside milling, cutting trajectory steering spindle rapid deceleration, in processing the Caton by the workpiece surface leave in a knife marks, the impact of thin-walled parts surface accuracy. For this problem, it is reasonable to use the line cutting path.

In the end, the optimization of the cutting line suddenly turned to avoid walking knife to make a hard bend, reduce the cutting direction of the cutting direction of the mutation. Programming software in general turning way to a right angle, when the cutting tool to the corner, cutting force and cutting direction will change, not only reduces the surface accuracy, but also seriously affect the service life of the tool. Therefore, in the processing of thin-walled parts, the turning point of the knife should be smooth and the surface machining precision is improved.

\section{Conclusion}

(1) This paper analyzes the advantages and disadvantages of the thin-walled parts processing scheme, and adopts the most appropriate processing method, which can reduce the machining deformation.

(2) The finite element analysis is carried out on the basis of the existing thin-walled parts clamping scheme, and adopt appropriate the method of minimum deformation and uniform distribution 
(3) Analysis of the cutting tool material and the role of each angle, the tool angle to take the corresponding value toimprove the tool life, while the surface accuracy of the workpiece has a certain guarantee.

(4) For the existing milling cutter route of thin-walled parts, that the the knife route is described for the optimal route, and the smoothness of the knife is optimized.

(5) The purpose of this paper is to improve the machining quality which is the surface of the aluminum alloy thin-walled parts, and analyze the influence on the machining quality of the thin-walled parts from the aspects of machining process plan, the selection of the clamping scheme, the selection of cutting tools and cutting line. Have a good guide to the actual processing.

\section{Acknowledgements}

The research in this paper is supported by the Ministry of science and technology international cooperation project (2014DFA72990).

\section{References}

[1] J. Xiaohui, "Investigation on The Mechanism of The Residual Stress and Machaning Accuracy Control Method of Complex Thin-Walled Parts", D. Donghua University (2014)

[2] P. Changlan, L.Weiwu, L. Zhiyong, "Improvement Measures of NC Machining Quality for Aircraft Aluminum Alloy Thin-walled Webs", J.Manufacturing Automation, vol.36, no.7, (2014), pp. 44-47.

[3] W. Shuhong, "Study on Initial Residual Stresses and Their Effects on Milling Distortion for Thick Aero-Aluminum-Alloy Plate”, D. Nanjing University of Aeronautics \& Astronautics, (2005)

[4] W. Yijiang, "Finite Element Simulation and Experimental Research on Deformation for Milling Process of Aluminium alloy Thin-walled Parts", D. North Central University.(2011)

[5] G. Xiang, W. Yong, "Research on the Precise NC Milling Technology of Thin-walled Workpieces", J. Machine Tool and hydraulic, vol.37, no.9, (2009), pp.14-17.

[6] W. Shaohong, "Research on Deformation in End Milling of Aerospace Thin-walled Structure Part", D. Dalian Jiaotong University (2008).

[7] N. Weigen, "The Thin-Walled Workpiece with High Speed Milling Path Optimization and Tooling Design", D. Nanjing University of Science and Technology, (2007).

[8] J. Wei, "Study on Error Prediction for Thin-walled Workpiece Milling Process and Developing Technology of Fast Milling Simulating Platform”, D. Nanchang Aviation University, (2012).

[9] G. Hun, Z. Dunwen, W. Shuhong, X. Lilin, W. Min, "Effect of Tool-path on Milling Accuracy under Clamping", J. Transactions of Nanjing University of Aeronautics\& Astronautics, vol.22, no.3, ( 2005), pp.234-239 